\title{
Sequential hemorrhage in two lesions of multiple cerebral cavernous malformations: A case report
}

\author{
Dan-Sorin Buzoianu', Maria-Irina Munteanu', Armand Frasineanu', \\ Radu-Dan Mihailescu' ${ }^{1}$, Georgiana Marin ${ }^{1}$, Alina-Lucia Ciuciulete ${ }^{1}$, Iuliana Cocianga ${ }^{1}$, \\ Bogdan Ovidiu Popescu ${ }^{1,2}$ \\ ${ }^{1}$ Department of Neurology, "Colentina" Clinical Hospital, Bucharest, Romania \\ 2"Carol Davila" University of Medicine and Pharmacy, Bucharest, Romania
}

\begin{abstract}
We present the case of a 64-year old female, with a 5 year history of symptomatic multiple cerebral cavernomas, who developed paresthesia in the right leg and arm, followed by vertigo, slurred speech and forward gaze diplopia. Brain investigation revealed two lesions with supra- and infratentorial localization with signs of recent hemorrhage of different ages. The localization of the lesions in the midpons and the basal ganglia imposed a conservative approach.
\end{abstract}

Keywords: multiple cerebral cavernous malformations, hemorrhage, brainstem cavernoma

\section{INTRODUCTION}

Cavernous malformations are slow-flow anomalies characterized by clustered vascular cavities consisting of enlarged capillaries, lined only by a thin endothelium and few subendothelial cells, without smooth muscle or elastic tissue, embedded in a collagen matrix without neural tissue producing a mulberry-like appearance (1).

They represent approximately $15 \%$ of all cerebral malformations (2). There are sporadic and familial variants with estimated prevalence in the general population as $0.1-0.5 \%$, the latter being autosomal dominant with incomplete penetrance and representing about $10-40 \%$ of the cases (3). The sporadic form usually results in a single malformation, whereas the familial form is associated with multiple cavernomas and mutations in 3 genes: CCM1 (encodes KRIT1 protein), CCM2 (encodes MGC4607 protein) and CCM3 (encodes PDCD 10 protein) with more than 150 distinct mutations described, the proportions linked to each CCM locus were $40 \%$ CCM1, 20\% CCM2 and 40\% CCM3 (4).
The neurological manifestations described include focal or generalized seizures (40-70\%), focal symptoms (35-50\%), cerebral hemorrhage (30$40 \%$ ) and headache (10-30\%) (5).

The investigation tool for diagnosis and follow-up is MRI, which differentiates between 4 types of lesions (6): acute form type 1 (hyperintense signal T1 and T2 sequences), mixed acute and chronic lesion type 2 (mixed hyper/hypointense signal T1 and T2 sequences), chronic usually asymptomatic type 3 (hypointense signal T1, T2 and gradient-echo sequences) and type 4 (hypointense signal only on gradient-echo sequence). The latter is strongly connected with familial forms (7).

Conservative or surgical treatment should be considered depending on the localization, intensity of the symptoms or their progression.

\section{CASE REPORT}

A 64-year old female was referred to our clinic with vertigo, slurred speech and forward gaze diplopia, preceded, two days before, by sudden right 
leg and arm paresthesia. Five years ago she underwent a cerebral MRI investigation for chronic left side headache, which revealed multiple lesions, both infra and supratentorial, suggestive of multiple cerebral cavernous malformations. She had a history of hypertension and hypercholesterolemia. There was no family history of hemorrhagic stroke or cerebral malformations.

The cerebral computed tomography examination performed before admission in our hospital showed diffuse hyperdense lesions located in the pons, left frontal lobe and right occipital lobe.

The neurological examination on admission revealed left abducens palsy, left peripheral facial palsy, right arm weakness $4-/ 5$, right limbs ataxia, hypoesthesia on both right arm and leg, Babinski sign on the right and dysarthria. Her blood pressure was $160 / 80 \mathrm{mmHg}$. She reported high blood pressure values in the past two weeks, although being under treatment.

Her clinical condition continued to deteriorate as she developed right leg 3/5 weakness.

The MRI investigation indicated multiple lesions located in the pons, right occipital lobe, left antero-lateral wall of the third ventricle and bilateral fronto-parietal lobes. Two of them, both approximately $2 / 2 \mathrm{~cm}$ in size, one in the pons and the other on the left antero-lateral wall of the third ventricle, showed T1W (Fig. 1), T2W (Fig. 2, 3)

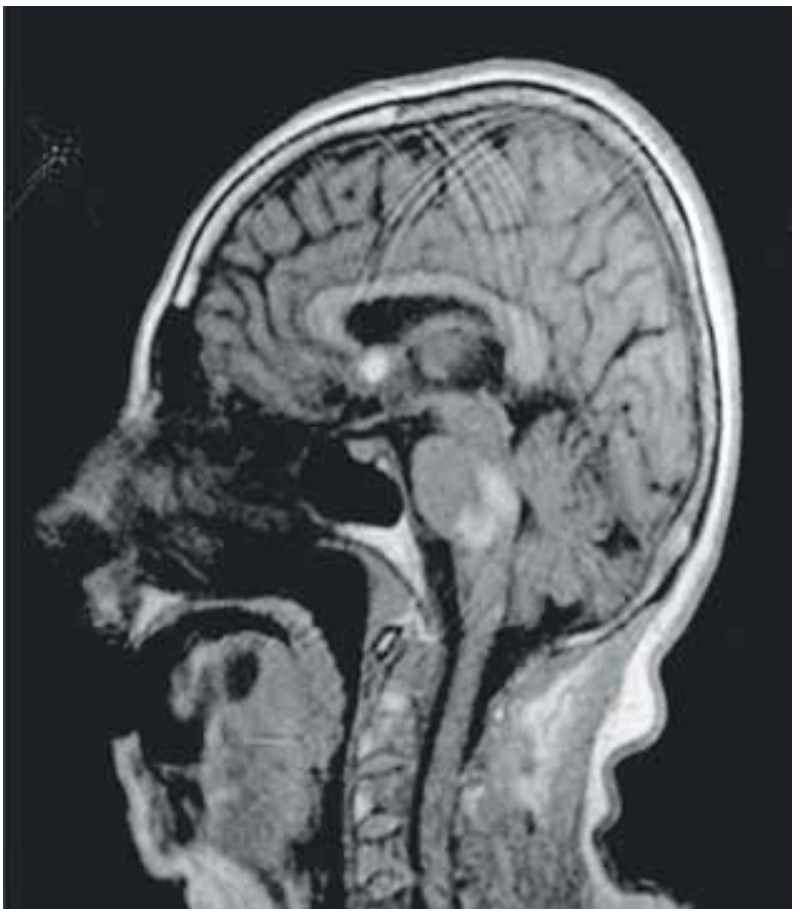

FIGURE 1. Sagital T1W showing two hyperintense lesions (pons and left lateral wall of third ventricle)

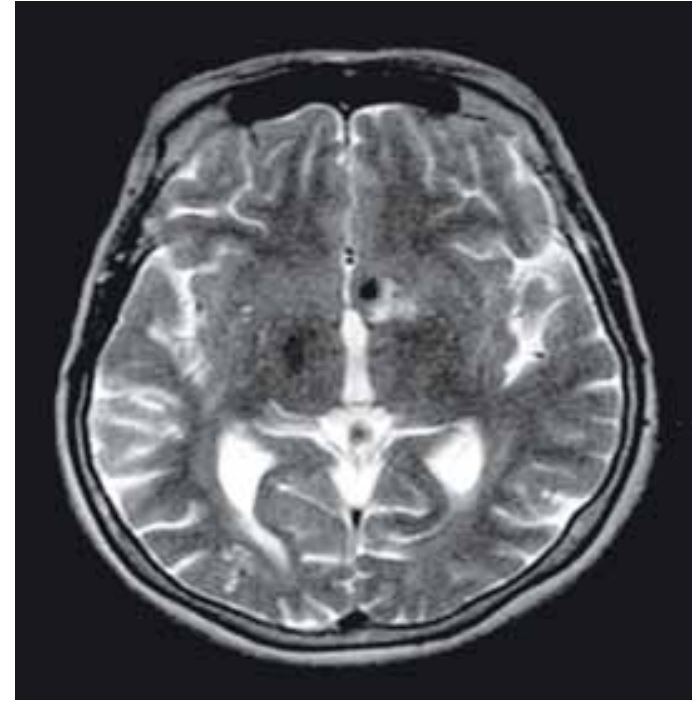

FIGURE 2. Transversal T2W sequence showing two lesions (left antero-lateral wall of third ventricle and right occipital lobe)

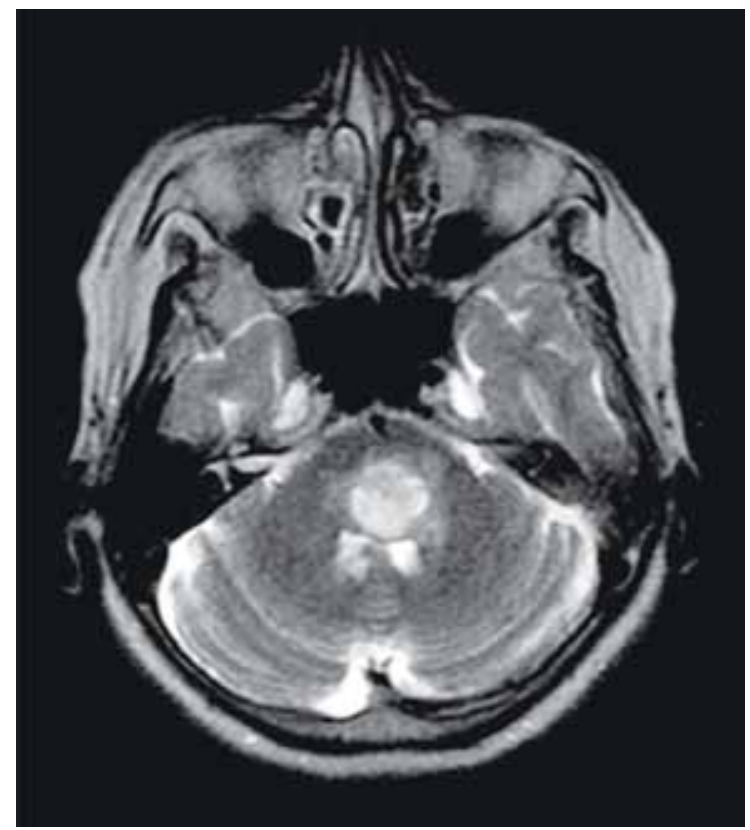

FIGURE 3. Transversal T2W sequence showing hyperintense lesion in the pons

and FLAIR (Fig. 4, 5) hyperintense signal, increased signal on diffusion weighted imaging (Fig. $6,7)$, and modified signal on the $\mathrm{T} 2 *$ gradient echo sequence (Fig. 8,9) with surrounding edema suggesting signs of recent hemorrhage. However there seems to be a chronological difference between the two events by comparing the DWI and T2*GRE sequences: acute bleeding in the lesion of the pons (high signal T2*GRE and restricted DWI signal) and subacute bleeding in the lesion on the antero-lateral wall of the third ventricle (mixed low/ high signal T2*GRE and restricted DWI signal). 


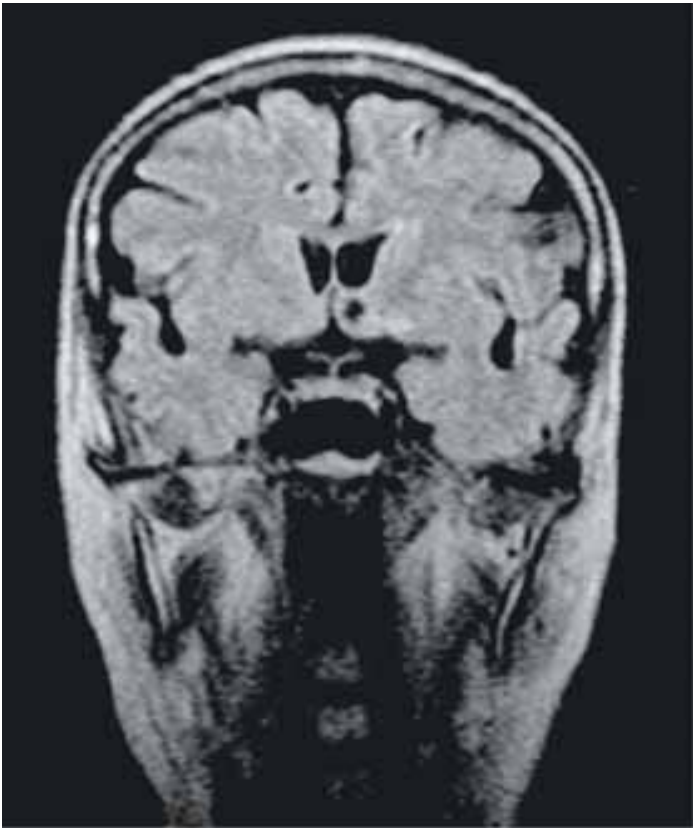

FIGURE 4. Coronal FLAIR sequence showing hypointense lesion with peripheral hyperintensity left antero-lateral wall of third ventricle

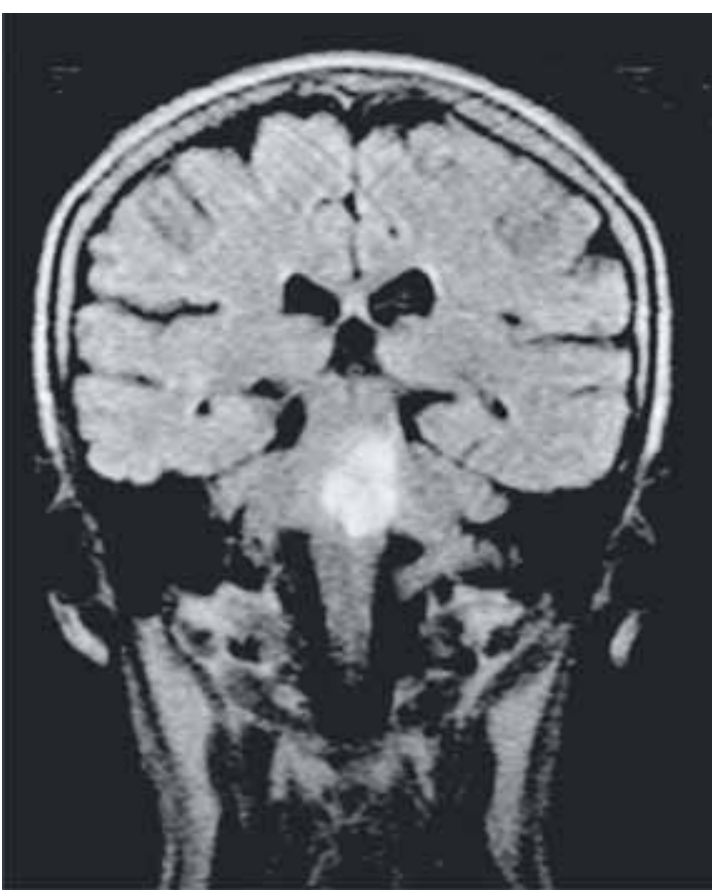

FIGURE 5. Coronal FLAIR sequence showing hypo-hyperintense lesion in the pons

The patient received conservative treatment due to the deep localization of the acute bleeding and was discharged with left Millard-Gubler syndrome and right face hypoesthesia.

\section{DISCUSSION}

The discovery of multiple cerebral cavernous malformations is made in up to $18.9 \%$ of the cases (8). Approximately 75\% (9) of the patients with

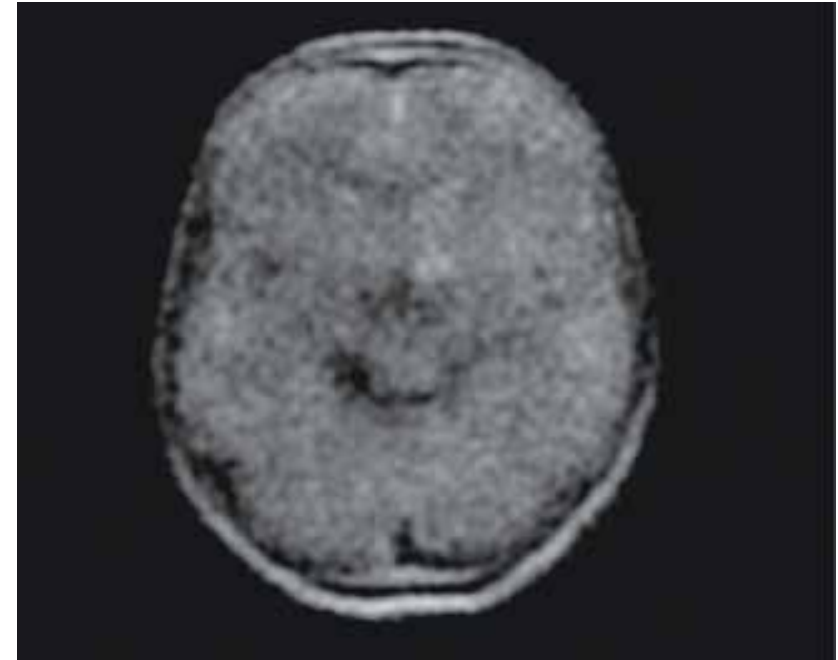

FIGURE 6. Increased signal left antero-lateral wall of third ventricle on DWI sequence

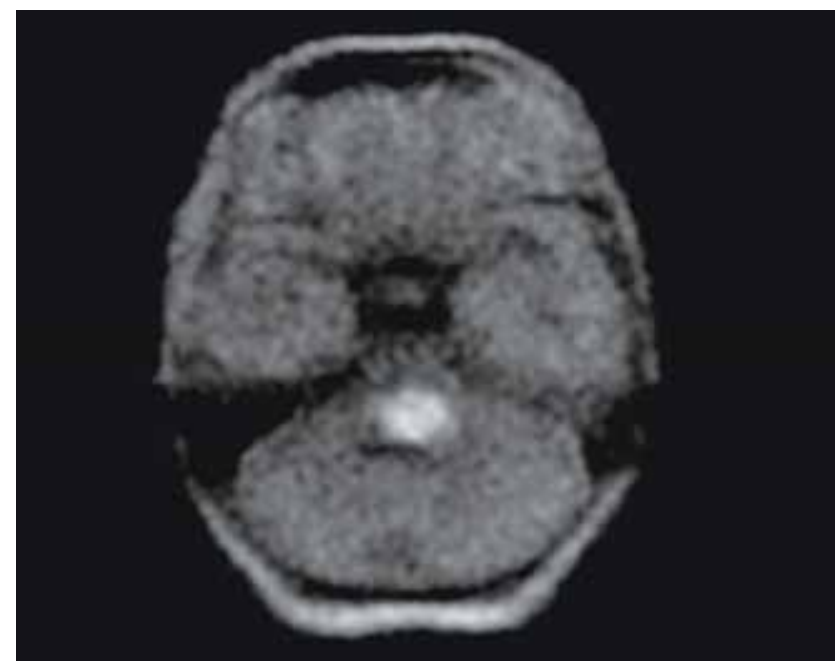

FIGURE 7. Increased signal pons on DWI sequence

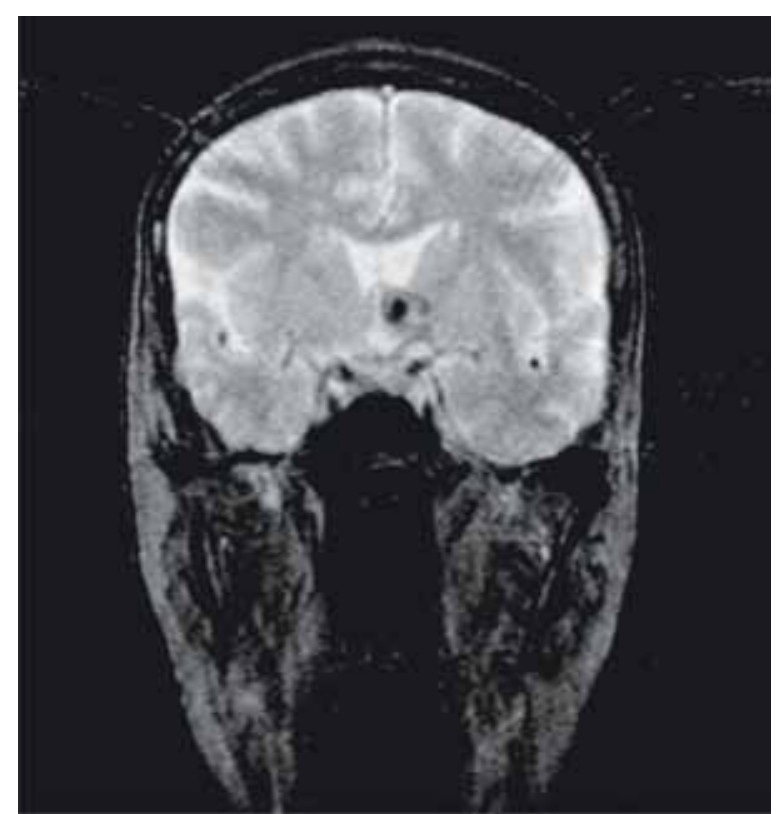

FIGURE 8. Coronal T2* gradient echo sequence sequence showing hypointense with surrounding hypointense lesion antero-lateral wall of the third ventricle 


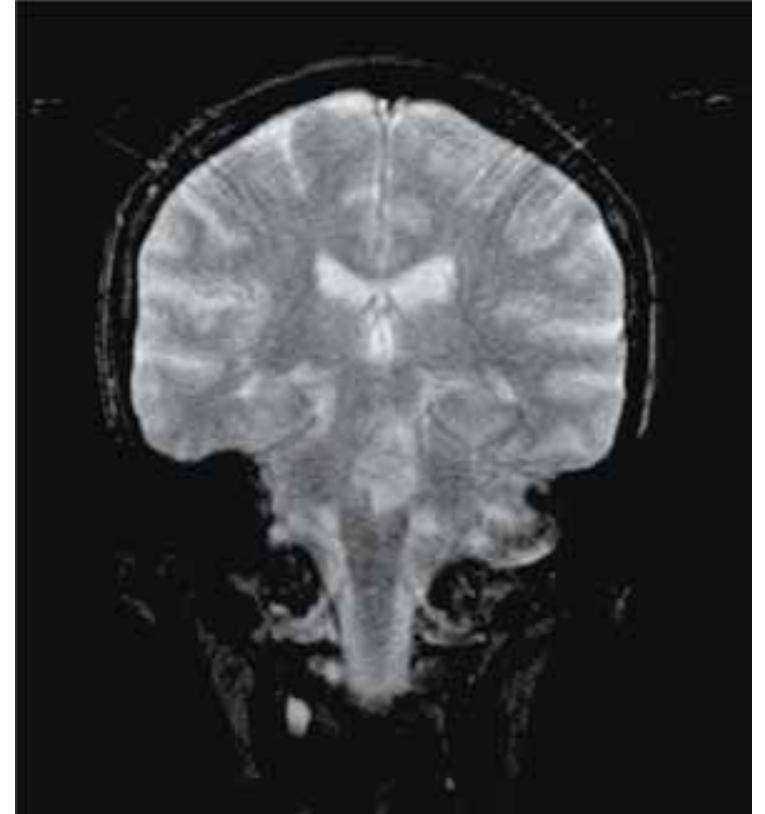

FIGURE 9. Coronal T2* gradient echo sequence sequence showing hyperintense lesion in the pons

multiple cavernomas have found affected relatives. The risk is even higher if the patient is symptomatic and the number of lesions exceeds five7, therefore genetic testing and MRI of the patient would be advised. Although without a prior family history of cerebral cavernoma, our case presented more than 5 lesions, both supra- and infratentorial, which is highly suggestive of a familial form. As follows we recommend genetic testing of the patient and in the situation of uncovering a familial variant, the screening should be extended to the family members.

The location dominance of the lesions is supratentorial $(86 \%$ of the malformations are localized above the tentorium cerebelli), the distribution being roughly the same distribution as the nervous tissue, seemingly without differences in likelihood of hemorrhage between their location (10).

Simultaneous hemorrhage of multiple cerebral cavernous malformations is a rare finding; to the date we have identified a number of four such reports (11-14). Simultaneous infra- and supratentorial hemorrhaging was reported in only one situation11. In our case the MRI would suggest sequential recent hemorrhages from two different cavernomas, the first event (hemorrhage of the lesion on the left antero-lateral wall of the third ventricle) induced no symptomatology, as the patient referred to the hospital with signs and symptoms suggestive only of a brainstem event.
Concerning the risk of cerebral hemorrhage, the risk in asymptomatic cases is different between the sporadic form group ( $0.6 \%$ per year) (15) and the familial form group (4.3\% per year) (16). The overall rates of annual hemorrhage is $1.6-3.1 \%$; regarding specific types: incidental discoveries 0.08 $0.2 \%$, unruptured cavernomas $0.3-0.6 \%$ and ruptured cavernomas $4.5-22.9 \%$ - the rates within the first year were found to be $14-18 \%$ (17).

The strongest risk factor associated with rebleeding was prior symptomatic hemorrhage, with a median time between the first and the second hemorrhagic event of 8 months (18). Other possible risk factors were brainstem localization and female gender; it is useful to mention that lesion size, multiplicity and antiplatelet therapy does not impact the risk for hemorrhage (17) 17.

The therapeutic options lay between observation and surgical intervention. The gold standard treatment for symptomatic and surgically-accessible cavernomas is the surgical resection. The incidental discoveries and the surgical inaccessible cavernous malformations are usually left under observation. In our case the localization of the hemorrhagic lesions in the brainstem and the basal ganglia imposed a conservative attitude. A new controversial method of treatment could be stereotatic surgery but at this time there is little evidence to support it (19).

\section{CONCLUSIONS}

Summing up the above, the patient presented recent hemorrhage in two cavernous malformation, only one symptomatic. Though not simultaneous, the brain imaging suggests a close time gap between the two events.

Due to the surgically unaccessible localization of the lesions (midpons and basal ganglia) the surgical intervention was not an option.

The risk of rebleeding is very high considering the risk factors: symptomatic event, brainstem localization and female gender, so close monitoring is mandatory.

The number and the localization of the cavernomas is highly suggestive of a familial form, therefore we recommend genetic testing of the patient and in the occurrence of a familial variant, of the relatives. 


\section{REFERENCES}

1. Cavalcanti D.D., Kalani Y.S., Martirosyan N.L. et al. Cerebral cavernous malformations: from genes to proteins to disease. Journal of Neurosurgery. 2012 Jan; 116.1:122-132.

2. Rigamonti D., Drayer B.P., Johnson P.C. et al. Cerebral cavernous malformations. Incidence and familial occurence. N Engl J Med. 1998; 319,343-347.

3. Labauge P., Denier C., Bergametti F. et al. Genetics of cavernous angiomas. Lancet Neurol. 2007 Mar 6;(3),237-244.

4. Riant F., Bergametti F., Ayrignac X. et al. Recent insights into cerebral cavernous malformations: the molecular genetics of CCM. FEBS Journal. 2010; 277:1070-1075.

5. Kumar A., Bhandari A., Goswami C. Surveying genetic variants and molecular phylogeny of cerebral cavernous malformation gene, CCM3/PDCD10. Biochem Biophys Res Commun. 2014 Dec 5;455(1-2):98-106.

6. Zabramski J.M., Wascher T.M., Spetzler R.F. et al. The natural history of familial cavernous malformations: results of an ongoing study. J Neurosurg. 1994; 80:422-32.

7. Brunereau L., Labauge P., Tournier-lasserve E. et al. Familial form of intracranial cavernous angioma: MR imaging findings in 51 families. Radiology. 2000; 214(1):209-16.

8. El-Koussy M., Stepper F., Spreng A. et al. Incidence, clinical presentation and imaging findings of cavernous malformations of the CNS. A twenty-year experience. Swiss Med Wkly. 2011 Apr; 13.141:w13172.

9. Labauge P., Laberge S., Brunereau L. et al. Hereditary cerebral cavernous angiomas: clinical and genetic features in 57 French families. Lancet. 1998; 352(9144): 1892-7.
10. Del Curling J.O., Kelly D.L. Jr, Elster A.D. et al. An analysis of the natural history of cavernous angiomas. J Neurosurg. 1991;75:702-8.

11. Chanda A., Nanda A. Multiple cavernomas of brain presenting with simultaneous hemorrhage in two lesions: a case report. Surg Neurol. 2002; 57(5):340-4.

12. Panciani P.P., Agnoletti A., Fornaro R. et al. Multiple cavernomas of the brain: simultaneous hemorrhage of two lesions in a non-familial form. Turk Neurosurg. 2012;22:671-4.

13. El Asri A.C., Derraz S., Gazzaz M. et al. Simultaneous bleeding of supratentorial and infratentorial cavernomas. Headache. 2015; 55(5):680-1.

14. Louis N., Marsh R. Simultaneous and sequential hemorrhage of multiple cerebral cavernous malformations: a case report. Journal of Medical Case Reports. 2016;10:36.

15. Labauge P., Brunereau L., Laberge S. et al. Prospective follow-up of 33 asymptomatic patients with familial cerebral cavernous malformations. Neurology. 2001; 57:1825-1828.

16. Kondziolka D., Lunsford L.D., Kestle J.R. The natural history of cerebral cavernous malformations. J Neurosurg. 1995;83:820-824.

17. Gross B.A., Du R. Cerebral cavernous malformations: natural history and clinical management. Expert Rev Neurother. 2015;15(7):771-7.

18. Flemming K.D. et al. Prospective hemorrhage risk of intracerebral cavernous malformations. Neurology. 2012;78.9:632-636.

19. Nagy G., Kemeny A.A. Radiosurgery for cerebral cavernomas. $J$ Neurosurg Sci. 2015 Sep;59(3):295-306.

Conflict of interest: none declared Financial support: none declared 